\title{
ANÁLISE DA FREQUÊNCIA DOS DIAGNÓSTICOS HISTOPATOLÓGICOS DAS NEOPLASIAS INTRAEPITELIAS CERVICAIS CONFORME A FAIXA ETÁRIA EM HOSPITAL DE REFERÊNCIA REGIONAL
}

\author{
ANALYSIS OF THE FREQUENCY OF HISTOPATHOLOGICAL DIAGNOSIS OF CERVICAL
} INTRAEPITEL NEOPLASMS ACCORDING TO THE AGE RANGE IN A REGIONAL REFERENCE

\section{HOSPITAL}

\author{
Abdalla Dib Chacur'; Antônio Mateus Henriques Nunes ${ }^{2}$ \\ 1 Professor Adjunto do Departamento de Ginecologia e Obstetrícia da Faculdade De Medicina de Campos - \\ FMC. Campos Dos Goytacazes, RJ- Brasil \\ ${ }^{2}$ Acadêmico do 9o período da Faculdade De Medicina De Campos - FMC. Campos Dos Goytacazes, RJ- Brasil
}

Faculdade De Medicina De Campos: Avenida Doutor Alberto Torres 217 - TÉRREO, 28035-580 CAMPOS DOS GOYTACAZES - RJ

Hospital Escola Álvaro Alvim: R. Barão da Lagoa Dourada, 409 - Centro, Campos dos Goytacazes - RJ, 28035-211 Autor correspondente: Antônio Mateus Henriques Nunes, Campos dos Goytacazes, 28035-053, Parque Tamandaré, № 245, Bloco 3, Apartamento 704.

\section{RESUMO}

O câncer de colo uterino representa um grande problema de saúde pública. Em geral, a evolução do câncer do colo do útero é lenta, passando por fases pré-clínicas detectáveis e curáveis. Os programas de rastreamento para as lesões precursoras (neoplasias intraepiteliais cervicais) se baseiam na utilização do exame citopatológico (preventivo), também conhecido como exame de Papanicolau. 0 objetivo deste estudo foi verificar a frequência dos diagnósticos histopatológicos de neoplasias intraepiteilais cervicais de baixo risco (NIC 1) e de alto risco (NIC 2 e 3), conforme as faixas etárias, em hospital de referência regional do norte fluminense no período de 2014 a 2016, verificando se as recomendações do Ministério da Saúde refletem nos dados epidemiológicos encontrados na nossa região. Tratase de uma pesquisa de abordagem quali-quantitativa envolvendo 352 amostras. Os dados foram analisados estatisticamente por meio do Software EPIDATA versão 3.0. Os resultados demonstram que os maiores percentuais de diagnósticos obtidos de lesões precursoras, tanto da lesão de baixo risco quanto de alto risco, ocorreram na faixa etária de 25 a 35 anos, com declínio progressivo destes diagnósticos à medida em que as faixas etárias aumentaram.

Palavras Chaves: Câncer de colo de útero. Lesões precursoras. Rastreio.

\section{ABSTRACT}

Cervical cancer represents a major public health problem. In general, the evolution of cervical cancer is slow, going through detectable and curable preclinical phases. Screening programs for precursor lesions (cervical intraepithelial neoplasms) are based on the use of cytopathological (preventive) examination, also known as Pap smear. The aim of this study was to verify the frequency of histopathological diagnoses of low-risk (CIN 1) and high-risk (CIN 2 and 3 ) cervical intraepithelial neoplasias, according to age groups, in a regional referral hospital in northern Rio de Janeiro in 2014. 2016, checking whether the recommendations of the Ministry of Health reflect the epidemiological data found in our region. This is a qualitative and quantitative research involving 352 samples. Data were statistically analyzed using EPIDATA Software version 3.0. The results show that the highest percentages of diagnoses obtained from precursor lesions, both low-risk and high-risk, occurred in the 25-35 age group, with progressive decline in these diagnoses as the age groups increased.

Keywords: Cervical cancer. Precursor lesions. Screening. 
O câncer de colo uterino representa um grande problema de saúde pública. Nos países em desenvolvimento possuiu elevada incidência e morbidade. Estima-se que 9 de 10 óbitos ocorridos no mundo por câncer do colo uterino situam-se em regiões menos desenvolvidas'. No Brasil, para o biênio 2018-2019, são esperados cerca de 16.370 novos casos de câncer do colo de útero².

Em 1968, Richart e Barron propuseram o termo Neoplasia Intraepitelial Cervical (NIC) para se referir às lesões histológicas do epitélio escamoso cervical, que se iniciariam como uma lesão de displasia leve e culminariam em carcinoma invasor, considerando tais lesões como um fenômeno único, contínuo e progressivo. Dessa forma, as neoplasias intraepiteliais cervicais foram divididas em três grupos de acordo com o comprometimento do epitélio cervical, podendo ser NIC 1, 2 ou 3, correspondendo respectivamente às lesões antes designadas como displasia leve, moderada e acentuada/carcinoma in situ. Contudo, essa premissa foi reavaliada por estudos subsequentes que mostraram que a maioria das mulheres com NIC 2 e 3 não apresentaram necessariamente lesões anteriores de $\mathrm{NIC} 1^{3}, 4$.

Em 1988, o Sistema Bethesda foi idealizado por especialistas reunidos em Maryland (USA). Propuseram nova terminologia citopatológica, dividindo as atipias citológicas identificadas pelo preventivo em duas categorias: as lesões intraepiteliais escamosas de baixo grau, que corresponde à definição de NIC I, e as lesões intraepiteliais de alto grau, que agrega as lesões consideradas como NIC II e III6,7.

As lesões precursoras do câncer do colo uterino possuem como substrato etiopatogênico a infecção persistente pelo vírus HPV, especialmente os subtipos 16 e 18, que apresentam maior potencial oncogênico. Ao penetrar na célula metaplásica imatura, resultante do processo fisiológico de metaplasia escamosa, o vírus perde seu capsídeo, o que permite a liberação de suas proteínas. Estas interagem com moléculas citoplasmáticas e no núcleo com o DNA da célula hospedeira, o que determina o dano celular que resulta em atipias fenotípicas.

As lesões de baixo grau (NIC 1), ainda que associadas a infecção por HPV, apresentam uma alta probabilidade de regressão. Enquanto as lesões de alto grau (NIC 2 e 3) mostram comportamento preponderantemente evolutivo para o câncer invasor e, portanto, representam as verdadeiras lesões precursoras do câncer do colo de útero6,7.

O Ministério da Saúde recomenda o exame citopatológico em mulheres assintomáticas com idade entre 25 e 64 anos, a cada três anos, após haver sido verificado dois exames anuais consecutivos normais.

Nesse sentido, o objetivo deste estudo foi verificar a frequência dos diagnósticos histopatológicos de neoplasias intraepiteilais cervicais de baixo grau (NIC 1) e de alto grau (NIC 2 e $3)$, conforme as faixas etárias, em hospital de referência regional do norte fluminense no período de 2014 a 2016.

\section{MATERIALE MÉTODO}

Foram realizados levantamentos dos resultados histopatológicos de 352 mulheres com diagnósticos histopatológico de NIC 1,2 e 3, realizados pelo Serviço de Anatomia Patológica do Hospital Escola Álvaro Alvim, nosocômio de referência regional no norte fluminense, no período de 2014 a 2016.

As amostras histopatológicas foram obtidas por meio de biópsia ambulatorial do colo uterino ou por conização cervical, tanto pela técnica clássica quanto pela cirurgia de alta frequência (CAF). Considerou-se como lesão de baixo risco os diagnósticos de NIC 1 e de alto risco, NIC 2 e 3.

Os diagnósticos foram agrupados nas seguintes faixas etárias: até 24 anos, 25 a 35, 36 a 45, 46 a 55, 56 a 64 e mais de 65 anos. Os resultados foram analisados estatisticamente por meio do Software EPIDATA versão 3.0.

\section{RESULTADOS}

No período analisado foram coletados 352 casos, sendo 138 em 2014, 122 em 2015 e 92 em 2016. Do total de casos, 132 foram resultantes de biópsias de colo realizadas após as respectivas colposcopias e 220 oriundas de conizações cervicais.

Dos 352 casos analisados, 63 foram de diagnóstico de NIC 1 e 289 de NIC 2 ou 3, mostrando a elevada predominância do diagnóstico de lesões de alto grau, representando respectivamente, $18 \mathrm{e}$ $82 \%$ dos casos.

Dentre os diagnósticos de lesão de baixo risco (NIC 1), foram verificadas as seguintes distribuições percentuais, conforme as faixas etárias consideradas neste estudo: até 24 anos (6,3\%); 25 a 35 (38,1\%); 36 a 45 (23,8\%); 46 a $55(19 \%) ; 56$ a 64 
$(4,8 \%)$ e acima de 65 (7,9\%). Já no que tange às lesões consideradas como de alto risco (NIC 2 e 3 ), os respectivos percentuais foram de 8,$7 ; 34,6 ; 24,6$; 19,$7 ; 7,3 ;$ e 5,2\% - Figura 1.

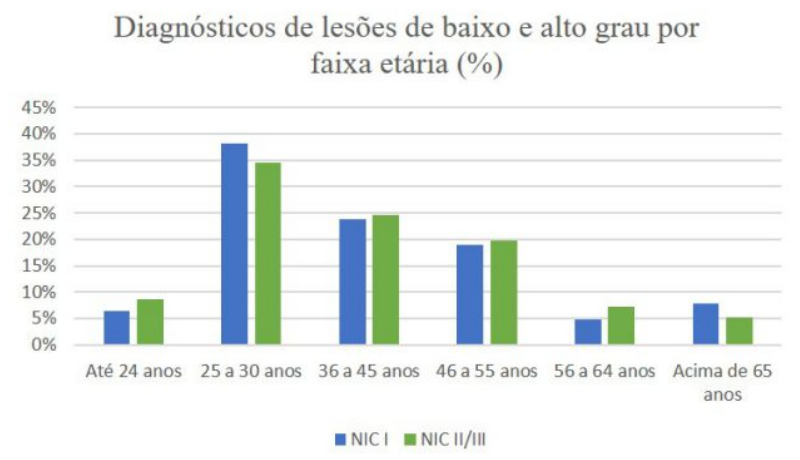

Figura 1: Diagnósticos de lesões de baixo e alto grau conforme faixa etária (\%)

\section{DISCUSSÃO}

Em geral, a evolução do câncer de colo uterino é lenta, passando por fases pré-clínicas detectáveis e curáveis. Os programas de rastreamento para as lesões precursoras (neoplasias intraepiteliais cervicais) se baseiam na utilização do exame citopatológico (preventivo), também conhecido como exame de Papanicolau.

O Ministério da Saúde recomenda o exame citopatológico de rastreio, isto é, de mulheres assintomáticas, com idades compreendidas entre 25 e 64 anos, a cada três anos, após dois exames anuais consecutivos normais. Em caso de resultado de lesão de baixo risco (NIC 1), a indicação é de repetição do exame em seis meses. Persistindo as alterações no exame citológico deve-se encaminhar a paciente para o exame colposcópico.

Caso o resultado citopatológico indique lesões de elevado risco (NIC 2 e 3), carcinoma in situ, carcinoma invasivo ou outras neoplasias malignas, deve-se encaminhar a paciente para o exame colposcópico e, na presença de lesão delimitada, retirar totalmente a lesão por cirurgia de alta frequência (CAF)8. Já a American Cancer Society em sua diretriz recomenda que as mulheres iniciem o panicolau a partir dos 21 anos9.

A detecção precoce do câncer do colo do útero em mulheres assintomáticas (rastreamento) permite a detecção das lesões precursoras da doença em estágios iniciais, antes mesmo do aparecimento dos sintomas. Esta estratégia representa uma ferramenta útil e eficaz de prevenção secundária do câncer de colo uterino, eis que mostra-se hábil para evitar cerca de $80 \%$ das mortes por este câncer8.

No presente estudo, observou-se um pico de incidência na faixa etária de 25 a 35 anos de lesões tanto de alto quanto de baixo risco, com declínio progressivo à medida em que as idades aumentaram. Esses dados refletem e corroboram as recomendações do Instituto Nacional de Câncer (INCA), expressadas nas Diretrizes Brasileiras para o Rastreamento do Câncer do Colo do Útero em 2016, nas quais se preconiza que o rastreamento citológico seja realizado em mulheres de 25 e 64 anos.

Entretanto, foram verificados que $6,3 \%$ das mulheres com menos de 25 anos apresentavam lesões de baixo risco e 8,7\% apresentavam lesões consideradas de alto risco. Ressalta-se que as mulheres dessa faixa etária (abaixo dos 25 anos) não estão incluídas nas recomendações para o rastreamento citológico do câncer de colo uterino do Brasil. Embora estas mulheres não estivessem na idade inicial preconizada pelo protocolo, o que poderia sugerir falha na atenção à saúde das mulheres, destaca-se que tais recomendações são relativas às políticas públicas de saúde e não ao atendimento realizado de forma privada.

Ademais, vários estudos reforçam o benefício do rastreio somente a partir dos 25 anos, como o que foi realizado pela International Agency for Research on Cancer (IARC) em 1986. Nesse estudo estimaram o risco do câncer de colo de útero em oito países com políticas públicas de rastreio diferentes. Concluíram que se o rastreamento se iniciasse a partir dos 20 anos de idade e não aos 25 , não geraria um grande impacto na redução da incidência do câncer de colo do útero e o início mais precoce diminuiria apenas $1 \%$ dos casos. Seus resultados demonstraram, ainda, que não existe uma diferença significativa na proteção oferecida ao utilizar o intervalo de 1 ano comparado ao intervalo de $3 / 3$ anos, enquanto a proteção oferecida foi menor quando se utilizaram o intervalo de $5 / 5$ anos10.

Um estudo feito na Inglaterra evidenciou que quando se inicia o rastreamento aos 20 anos de idade, determina aumento substancial de casos de sobretratamento e um modesto benefício ao rastreio. Pois, para prevenir um caso de carcinoma invasor do colo uterino seria necessário realizar de 12.500 a 40 mil exames adicionais em mulheres 
entre 20 e 24 anos e tratar somente 300 a 900 mulheres com NIC. Tais diagnósticos seriam necessariamente identificados mais adiante, sem prejuízos, considerando a evolução lenta dessas lesões11.

Ressalta-se, mais uma vez, que tais considerações devem ser analisadas à luz de políticas públicas de saúde e não em um cenário de atendimento médico privado, nos quais situações clínica-epidemiológicas exigem condutas individualizadas.

A triagem cervical em mulheres entre $20 \mathrm{e}$ 24 anos tem pouco ou nenhum impacto nas taxas de câncer cervical invasivo até os 30 anos11. Além disso, estatisticamente a incidência de câncer de colo de útero em mulheres com menos de 24 anos é sabidamente muito baixa e os procedimentos cirúrgicos para o tratamento das neoplasias intraepitelial cervicais em mulheres jovens estariam relacionados ao aumento de morbidade obstétrica e neonatal, como o abortamento e o parto prematuro12.

No total de casos identificados $(n=352)$, $13,1 \%$ dessas mulheres estavam acima dos 65 anos. Deste grupo, 7,9\% das mulheres apresentaram lesões de baixo grau e 5,2\% lesões de alto grau. Observa-se que, não existe um consenso nos estudos relacionados a idade máxima de rastreio do câncer do colo de útero13, ${ }^{1} 4$. Entretanto, com o crescente aumento da expectativa de vida da população feminina no Brasil14,15 faz-se necessária uma sistemática revisão deste limite visando atualização das recomendação para realização do exame de Papanicolau.

Com uma triagem negativa entre 50 e 64 anos, as mulheres apresentam uma diminuição de $84 \%$ no risco de desenvolver um carcinoma invasor entre 65 a 83 anos, em comparação as mulheres que não foram rastreadas 16.
Importa destacar que percentual não desprezível de casos de NIC 2 apresenta comportamento evolutivo incerto, cujas lesões tendem a retroceder ao longo do tempo caso sejam instituídas condutas expectantes de controle. Entretanto, os casos de NIC 3 apresenta uma grande chance de evolução para neoplasia invasora, o que i m põ e a realização de condutas terapêuticas17,18,19.

\section{CONCLUSÃO}

Os maiores percentuais de diagnósticos obtidos de lesões precursoras, tanto da lesão de baixo risco quanto de alto risco, ocorreram na faixa etária de 25 a 35 anos, com declínio progressivo destes diagnósticos à medida em que as faixas etárias aumentaram. Conclui-se que estes resultados corroboram as recomendações do Instituto Nacional de Câncer (INCA) expressadas nas Diretrizes Brasileiras para o Rastreamento do Câncer do Colo do Útero de 2016, por meio das quais se preconiza que o rastreamento citológico seja realizado em mulheres de 25 a 64 anos.

Apesar deste estudo ter verificado diagnósticos de NIC em mulheres abaixo de 25 anos, publicações relacionadas ao tema mostram que programas que utilizam rastreamento citopatológico iniciando em idade mais precoce (antes dos 25 anos) não são mais eficientes, pois não geram impacto significativo na diminuição da incidência do câncer invasor. Além disso, estatisticamente a incidência de câncer de colo de útero em mulheres com menos de 24 anos é sabidamente muito baixa e o tratamento cirúrgico de lesões precursoras em mulheres muito jovens pode, ainda que infrequente, determinar anormalidades uterinas que venham a comprometer a fertilidade e aumentar o risco de complicações obstétricas durante à assistência ao pré-natal.

\section{REFERÊNCIAS}

1- FERLAY, Jaques et al. Cancer incidence and mortality patterns in Europe: estimates for 40 countries in 2012.

European journal of cancer, v. 49, n. 6, p. 1374-1403, 2013.

2- BRASIL. Ministério da Saúde (MS). Instituto Nacional de Câncer (Inca)..Estimativa 2018 - Incidência de Câncer no Brasil. Disponível em https://www.inca.gov.br/sites/ufu.sti.inca.local/files//media/document//estimativa-incidenciade-cancer-no-brasil-2018.pdf. Acesso em 10 de maio de 2019.

3- REMMINK, Ans J. et al. The presence of persistent high-risk HPV genotypes in dysplastic cervical lesions is associated with progressive disease: natural history up to 36 months. International Journal of Cancer, v. 61, n. 3, p. 306-311, 1995.

4- BARRON, Bruce A.; RICHART, Ralph M. A statistical model of the natural history of cervical carcinoma based on a 
prospective study of 557 cases. Journal of the National Cancer Institute, v. 41, n. 6, p. 1343-1353, 1968.

5- ZEFERINO, Luiz Carlos et al. Duração da neoplasia intra-epitelial e do carcinoma invasor do colo uterino: estudo epidemiológico. Revista Brasileira de Ginecologia e Obstetrícia, 1998.

6- KUMAR, V.; ABBAS, A. K.; FAUSTO, N.; MITCHELL, R.N.; Robbins - Patologia Básica. Rio de Janeiro: Elsevier; 2008. p. 782-784.

7- MENDOZA, L. P. et al. Distribution of human papillomavirus genotypes in Paraguayan women according to the severity of the cervical lesion. Journal of medical virology, v. 83, n. 8, p. 1351-1357, 2011.

8- BRASIL. Ministério da Saúde (MS). Instituto Nacional de Câncer (Inca). Diretrizes brasileiras para o rastreamento do câncer do colo do útero. Rio de Janeiro: Inca; 2016. Disponível em:

https://www.inca.gov.br/publicacoes/livros/diretrizes-brasileiras-para-o-rastreamento-do-cancer-do-colo-do-utero. Acesso em 10 de junho de 2019.

9- SMITH, Robert A. et al. Cancer screening in the United States, 2013: a review of current American Cancer Society guidelines, current issues in cancer screening, and new guidance on cervical cancer screening and lung cancer screening. CA: a cancer journal for clinicians, v. 63, n. 2, p. 87-105, 2013.

10- IARC WORKING GROUP ON EVALUATION OF CERVICAL CANCER SCREENING PROGRAMMES. Screening for squamous cervical cancer: duration of low risk after negative results of cervical cytology and its implication for screening policies. British Medical Journal (Clinical Research Edition), p. 659-664, 1986.

11- CASTANON, Alejandra et al. Cervical screening at age 50-64 years and the risk of cervical cancer at age 65 years and older: population-based case control study. PLoS medicine, v. 11, n. 1, p. e1001585, 2014.

12- SASIENI, Peter; CASTANON, Alejandra; CUZICK, Jack. Effectiveness of cervical screening with age: population based case-control study of prospectively recorded data. Bmj, v. 339, p. b2968, 2009.

13- KYRGIOU, M. et al. Obstetric outcomes after conservative treatment for intraepithelial or early invasive cervical lesions: systematic review and meta-analysis. The Lancet, v. 367, n. 9509, p. 489-498, 2006.

14- ISIDEAN, Sandra D.; FRANCO, Eduardo L. Counterpoint: cervical cancer screening guidelines-approaching the golden age. American journal of epidemiology, v. 178, n. 7, p. 1023-1026, 2013.

15- RUSTAGI, Alison S.; KAMINENI, Aruna; WEISS, Noel S. Point: cervical cancer screening guidelines should consider observational data on screening efficacy in older women. American journal of epidemiology, v. 178, n. 7, p. 1020-

1022, 2013.

16- CAMARGOS, Mirela Castro Santos et al. Estimativas de expectativa de vida livre de incapacidade funcional para Brasil e Grandes Regiões, 1998 e 2013. Ciência \& Saúde Coletiva, v. 24, p. 737-747, 2019.

17- LUFT, Frank et al. Detection of integrated papillomavirus sequences by ligation-mediated PCR (DIPS-PCR) and molecular characterization in cervical cancer cells. International journal of cancer, v. 92, n. 1, p. 9-17, 2001. 18- BEREK, Jonathan. Berek \& Novak: tratado de ginecologia. In: Berek \& Novak: tratado de ginecologia. 2014. 19- ELIT, Laurie et al. Expectant management versus immediate treatment for low-grade cervical intraepithelial neoplasia: a randomized trial in Canada and Brazil. Cancer, v. 117, n. 7, p. 1438-1445, 2011. 